\title{
Spatial learning impairments in aged rats trained with a salient configuration of stimuli
}

\author{
MARY ANN PELLEYMOUNTER, MARY Y. SMITH, and MICHELA GALLAGHER \\ University of North Carolina, Chapel Hill, North Carolina
}

\begin{abstract}
Aged animals are impaired in many maze learning tasks, suggesting that they may have a spatial learning deficit. Alternatively, declining sensory/perceptual faculties in aged animals may impede their ability to use complex arrangements of stimuli that are necessary for spatial learning. To test this possibility, we conducted place learning in the Morris water maze in the presence of a very simple and salient configuration of stimuli that consisted of a black plexiglass sheet, subtending an $\operatorname{arc}$ of $110^{\circ}$ around the inside wall of the water maze. In Experiment 1, place learning in young rats was guided by this configuration of stimuli. In Experiment 2, a prominent placelearning deficit in aged rats was observed when animals were trained with this configuration. Thus, the place-learning impairment in aged animals may not be confined to perceptual deficits that decrease an animal's ability to use complex configurations of stimuli.
\end{abstract}

Aged animals are impaired in a wide variety of maze learning tasks. For example, old animals are slower than young animals to acquire radial-maze tasks (Barnes, Nadel, \& Honig, 1980; Davis, Idowu, \& Gibson, 1983; deToledo-Morrell, Morrell, \& Fleming, 1984; deToledoMorrell, Morrell, Fleming, \& Cohen, 1984; Gallagher, Bostock, \& King, 1985; Wallace, Krauter, \& Campbell, 1980). Furthermore, aged rats are impaired on a holeboard escape task (Barnes, 1979; Barnes \& McNaughton, 1985; Barnes et al., 1980) as well as on the Morris water maze (Biegon, Greenberger, \& Segal, 1986; Gage \& Bjorklund, 1986; Gage, Dunnett, \& Bjorklund, 1984; Rapp, Rosenberg, \& Gallagher, 1987). The impaired performance of aged animals is viewed in many of these studies as a declining capacity to acquire and/or use spatial information.

Although deficits on any of these tasks could reflect a spatial learning impairment, studies using the Morris water maze have sought to further analyze the behavior of aged animals. The analysis is accomplished by comparing acquisition of the "place" and "cue" versions of the task (Morris, 1981). In the water maze, the animal is motivated to locate a cylindrical escape platform that is either visible (cue learning) or camouflaged and submerged just below the surface of the water (place learning). During place training, the escape platform remains in a fixed position in the maze; rapid escape depends on the animal's ability to identify the platform's position with reference to a configuration of cues located in the surrounding room. Such distal stimuli usually consist of assorted objects positioned around the maze. In the case of

This work was supported by NIMH Grant MH39180 and KO2MH00406 to M.G. and an NIMH postdoctoral fellowship to M.A.P. The authors also wish to thank R. A. Rosenberg for technical assistance. Please address correspondence to Mary Ann Pelleymounter, Department of Psychology, Davie Hall 013A, University of North Carolina, Chapel Hill, NC 27514. cue learning, a simple and highly salient stimulus (a black platform that rises above the water's surface) is used by the rat as a guide to escape. The spatial location of the platform during cue training is not usually relevant to performance, because it is randomly moved to different positions in the maze across trials.

Invariably, aged animals are impaired on the placetraining version of this maze task (Biegon et al., 1986; Gage \& Bjorklund, 1986; Gage et al., 1984; Rapp et al., 1987). Aged animals that demonstrate a place-learning deficit do not, however, show a comparable cue-learning deficit. Following assessment of a place-learning deficit in a group of aged rats, Rapp et al. (1987) found that the same animals had no deficit during cue training when the visible platform was moved to a position that differed from that used in place training. The latter result is consistent with reports that aged rats are unimpaired on a number of two-cue visual discrimination tasks (Kay \& Sime, 1962; Lowy et al., 1985; Sarter \& Markowitsch, 1983). For example, Lowy et al. (1985) reported that 24-month-old rats acquired a simple black/white visual discrimination as readily as did 6-month-old rats. These results provide general support for the interpretation that spatial learning may be disproportionately affected by aging. At the same time, these results do not rule out the possibility that old animals have a sensory/perceptual impairment that impedes their effective use of the stimuli that compose the traditional extramaze environment. Indeed, the stimuli that are used in spatial learning experiments are usually more complex and may be less salient than the stimuli employed in visual discrimination tasks. Aged animals might thus be less proficient at place learning due to a more subtle sensory/perceptual impairment that impedes their capacity of processing the visual information that is typically available in spatial learning experiments.

In the following experiments, we tested the possibility that the basis of the place-learning impairment in aged 
animals is a deficit in the ability to perceive and/or effectively use the information provided in the traditional extramaze environment. First, we examined some of the characteristics of place learning in young animals when a simple and highly salient configuration of stimuli was arranged on the inside wall of the maze. We then compared the acquisition of place learning in young and aged rats in this environment.

\section{EXPERIMENT 1}

\section{Method}

Subjects. Ten experimentally naive Long-Evans hooded male rats (Charles River Laboratories) were subjects in Experiment 1. They were housed in single cages in a vivarium that was maintained on a 12-h light/dark cycle (lights on at 7 a.m.) and climate-controlled at $25^{\circ} \mathrm{C}$. All animals were permitted access to food (Wayne Lab Blox) and water ad lib. The rats were approximately 4 months of age at the time of testing.

Apparatus. The Morris water maze used is a circular galvanized steel watering tank $1.83 \mathrm{~m}$ in diameter and $0.58 \mathrm{~m}$ in height, with its interior surfaces painted white. During testing, the tank was filled to a depth of $35.5 \mathrm{~cm}$ with water that was clouded by the addition of $0.9 \mathrm{~kg}$ powdered milk. The water was maintained at $26^{\circ} \pm 1^{\circ} \mathrm{C}$. A white escape platform ( $10.2 \mathrm{~cm}$ in diameter and $34.5 \mathrm{~cm}$ in height) was centered in one of four quadrants of the maze. This escape platform was always submerged $1 \mathrm{~cm}$ below the water's surface. A $0.58 \times 1.75 \mathrm{~m}$ sheet of flexible black plexiglass was placed along the inner wall of the tank, subtending an arc of $110^{\circ}$. This arc was centered at either the $10^{\prime}$ 'clock or 7 o'clock position. The maze was surrounded by white muslin curtains that intersected at each quadrant for ease of access to the different entry points in the maze. A schematic drawing of the maze is shown in Figure 1. A Panasonic WV-241P camera with a 4-mm wide-angle lens was suspended above the center of the maze at all times. Sessions were videotaped with a JVC VHS videocassette recorder that was located immediately outside the door to the maze room. An RCA 12-in. monitor was located inside the maze room, but outside the curtained area, to aid the experimenter in locating the animal's position within the maze.

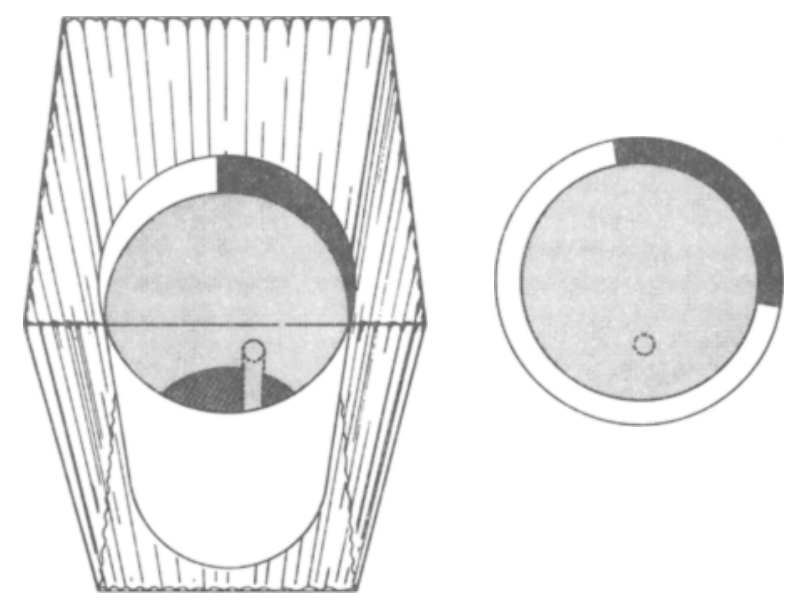

Figure 1. A schematic cut-away representation of the water maze is illustrated to the left. An overview of the same maze is shown to the right. Both views show the submerged escape platform used in place learning, along with the black Plexiglas arc. Curtains surround the maze.
Procedure. Prior to training, each animal was habituated to handling and to the maze itself. The animals were handled by the experimenter for 3 min per day for 1 week. On the day immediately preceding training, each animal was placed into the maze without the escape platform and allowed to swim for $90 \mathrm{sec}$. The animal was then returned to its home cage.

On each training trial the animal was placed into the maze, facing the tank wall at one of four equally spaced entry points; the entry point used on any given trial was varied. The animal was allowed to swim until it escaped onto the platform or until $120 \mathrm{sec}$ elapsed. If the animal had not found the platform by this time, it was placed onto the platform by the experimenter. The animal remained on the platform for $60 \mathrm{sec}$. The animal was then either placed in a holding cage for a 60 -sec intertrial interval or returned to its home cage at the end of a session. The subjects were given 6 training trials per day for 4 days. During Training Trials $1-17$, the submerged platform remained in the same location for each animal. Platform location, however, was varied among the animals. On Training Trials 19-23, both the arc and the platform location for each animal were rotated $180^{\circ}$ in the maze. Thus, on these trials, the relationship of the arc to the platform was maintained, but the items were in a different part of the maze.

Three probe trials were interpolated during training (Trials 12, 18 , and 24). During a probe trial, the escape platform was removed, and the animal was allowed to swim freely for $90 \mathrm{sec}$. During the second probe trial (Trial 18), the black arc was also removed from the maze. On the third probe trial, the position of the arc corresponded to its location on trials 19-23.

Videotapes of the probe trials were analyzed for several measures of spatial bias. Quadrant time was determined as the number of seconds that an animal spent in each of the four maze quadrants. The second measure, annulus crossings, refers to the number of times an animal traversed each of the four points at which a platform could be positioned.

Statistical Analysis. Escape latencies were analyzed using a oneway ANOVA. Quadrant times and annulus crossings were also analyzed using separate one-way ANOVAs for each of the three probe trials. Post hoc group mean comparisons were conducted using the Newman-Keuls statistic.

\section{Results}

As depicted in Figure 2, escape latencies decreased over the course of training, indicating that the animals learned to rapidly locate the escape platform. Analysis of escape latencies over all trials revealed a significant trials effect $[F(20,180)=9.38 ; p<.001]$.

Furthermore, when the escape platform was removed on the first probe trial (Trial 12), the animals showed a spatial bias for the quadrant that had formerly contained the escape platform. Analysis of quadrant times indicated that animals did not spend an equal amount of time in each quadrant $[F(3,36)=28.72 ; p<.001]$. This significant quadrant effect was due to a specific bias for the training quadrant $(p<.01)$. Analysis of the annulus-crossings measure yielded similar results. As was the case in the quadrant-time measure, there was a significant annulus effect $[F(3,36)=9.46 ; p<.001]$ that was due to a bias for the annulus that "marked" the former position of the escape platform $(p<.01)$.

The results of the second probe trial indicated that the spatial bias of animals in this task was not entirely dependent on the presence of the arc in the maze. Animals still exhibited a significant quadrant effect $[F(3,36)=$ 


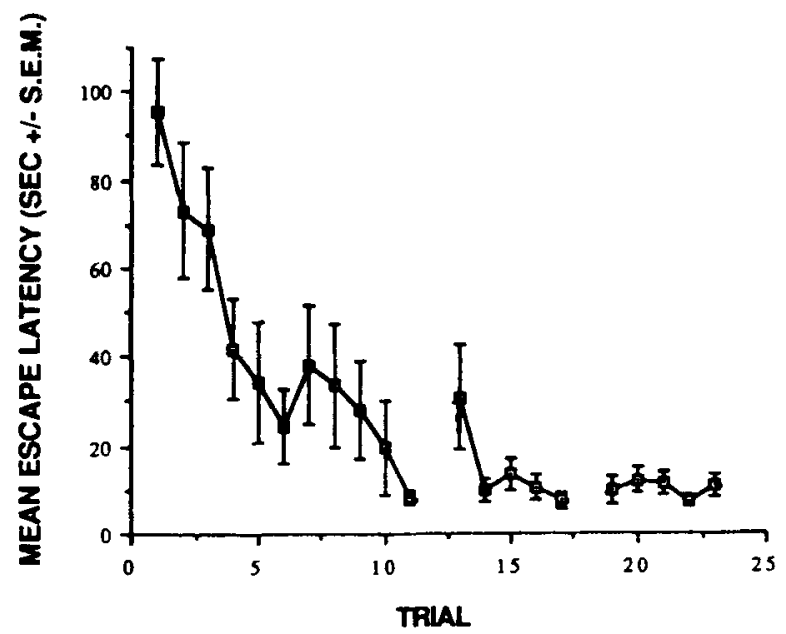

Figure 2. Mean escape latencies of young animals for Trials 1-23. Probe trials were interpolated on Trials 12, 18, and 24. These trials are indicated by the breaks in the graph. The platform and the arc were rotated $180^{\circ}$ on Trials $19-23$.

$5.93 ; p<.002$ ] that was attributable to a bias for the training quadrant $(p<.05)$. This was not the case for the annulus-crossings measure, because statistical analysis revealed no significant effect. Thus, when the arc was removed, there was no specific bias for the training annulus.

On the five trials (Trials 19-23) following the second probe trial, the arc and platform location for each animal were rotated $180^{\circ}$. As indicated in Figure 2, even on the first training trial after rotation (Trial 19) the rats rapidly escaped to the platform in the appropriate location relative to the arc's position. On the third probe trial, the arc remained in the new position but the platform was removed from the maze. The designated training quadrant and annulus corresponded to their locations on the five previous training trials. In results similar to those of the first probe trial, the animals showed a significant quadrant $[F(3,36)=19.64 ; p<.001]$ and annulus $[F(3,36)$ $=8.67 ; p<.001]$ bias. Again, these effects were due to a specific bias for the training quadrant $(p<.01)$ and annulus $(p<.01)$. Quadrant times and annulus crossings for the three probe trials are shown in Figures 3 and 4.

\section{Discussion}

The subjects in this experiment were able to learn the location of a hidden escape platform when a black arc on the inside wall of the maze provided a highly salient configuration of stimuli. Furthermore, they appeared to learn the location of the hidden platform relatively quickly, reaching asymptotic escape latencies $(9-11 \mathrm{sec})$ in 11 training trials. This acquisition rate is comparable to that found during place training with the same apparatus but using a more complex set of distal cues located outside the maze (see Figure 5).

The acquisition rate in the present experiment is also notably slower than that observed during cue training (see
Figure 5 for comparison). In addition, data from the first probe trial (Trial 12) indicated that the asymptotic escape latencies on Trial 11 reflected spatial learning because animals showed a strong spatial bias for the quadrant and annulus that had previously contained the escape platform.

This experiment was designed to examine whether the spatial behavior of these animals was dependent on, or at least guided by, the presence of the arc. Data from the second probe trial suggested that this was the case, at least for the annulus-crossings measure, which requires very

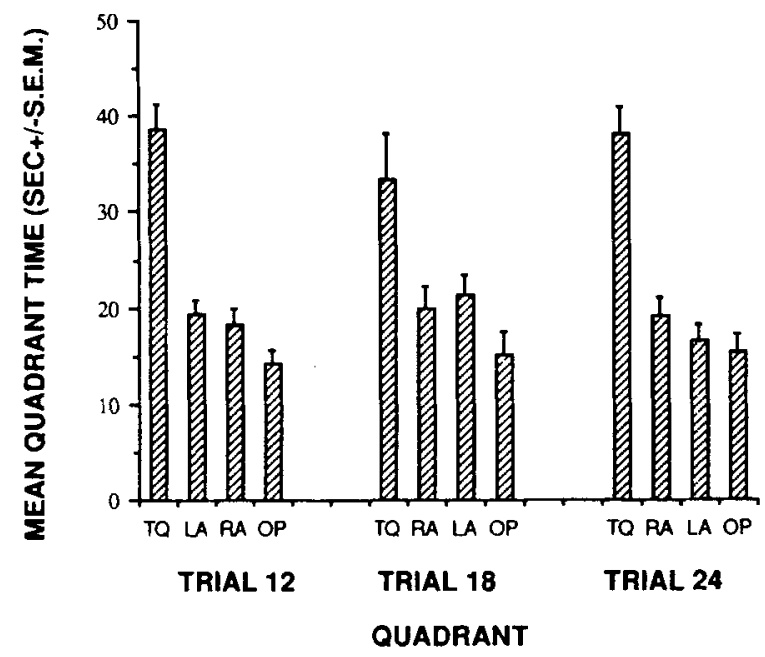

Figure 3. Mean quadrant time for Probe Trials 12, 18, and 24. The arc remained in its original position on Trial 12, was removed on Trial 18, and was rotated $180^{\circ}$ on Trial $24 . T Q=$ former training quadrant, $\mathbf{R A}=$ right adjacent, $\mathrm{LA}=$ left adjacent, and $\mathrm{OP}=$ quadrant exactly opposite $\left(180^{\circ}\right)$ the training quadrant.

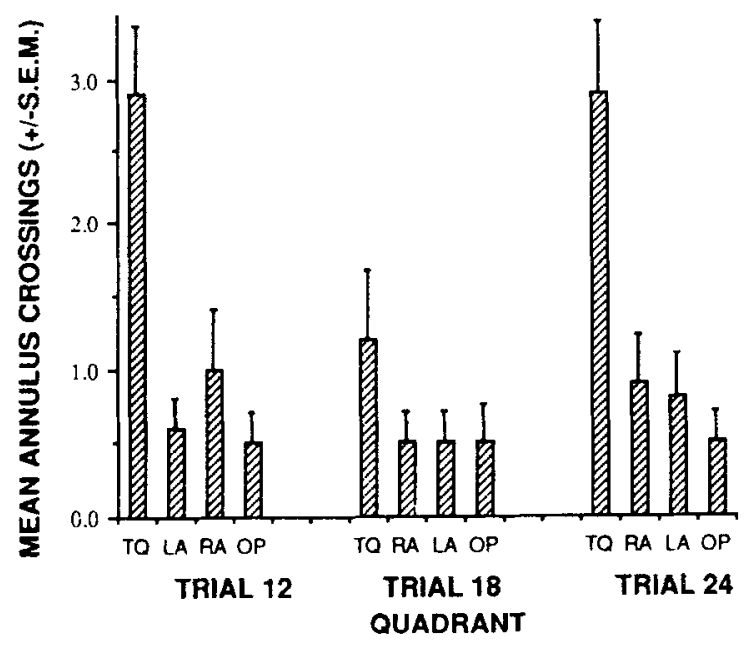

Figure 4. Mean number of annulus crossings for Probe Trials 12, 18, and 24. As in Figure 3, the arc remained in its original position on Trial 12, was removed on Trial 18, and was rotated $180^{\circ}$ on Trial 24. TQ = former training quadrant, $\mathbf{R A}=$ right adjacent, $\mathbf{L A}=$ left adjacent, and $\mathrm{OP}=$ quadrant opposite the training quadrant. 


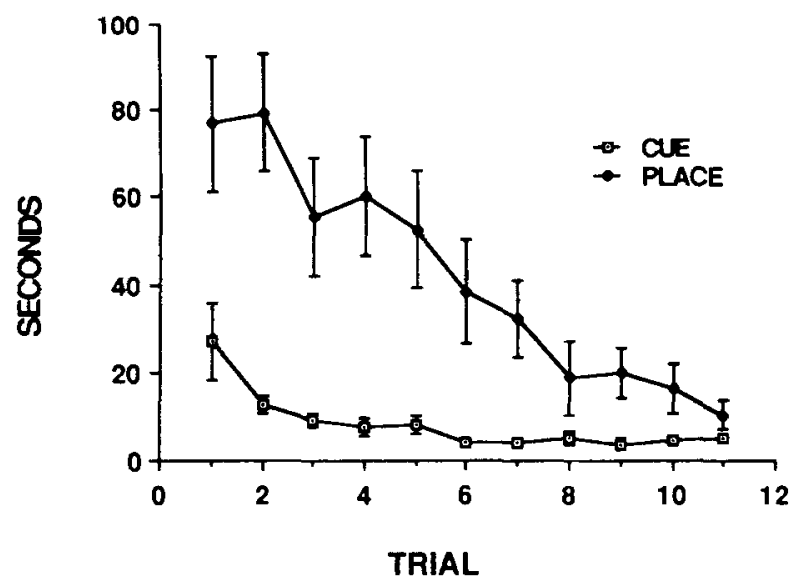

Figure 5. Mean escape latencies for young animals during cue and place training, using a traditional extramaze configuration of stimuli. A probe trial was interpolated on Trial 12 (Data are from Decker, Pelleymounter, \& Gallagher, in press).

precise localization in the maze. This was not the case for the quadrant-time measure, because the animals showed a residual bias for their former training quadrant even though the arc was not present. Therefore, the arc may not be necessary to locate the general region of the training quadrant, but may be important for the more precise localization of the platform's position.

When present, the arc/wall configuration did appear to be the dominant guide for spatial behavior, because the animals continued to accurately locate the platform when the arc and platform were rotated $180^{\circ}$. It is particularly important to note that, even on the first training trial after rotation occurred (Trial 19), the animals escaped rapidly (in less than $10 \mathrm{sec}$ ) by swimming directly to the appropriate location in the tank with respect to the arc. The spatial bias at this new location was also clearly evident during the last probe trial (Trial 24).

\section{EXPERIMENT 2}

In Experiment 2, we compared place learning in young and aged rats, using the arc/wall configuration.

\section{Method}

Subjects. Twelve young and 8 aged male Long-Evans hooded rats (Charles River Laboratories) were tested. Young rats were approximately 4 months old and aged rats were 24-26 months old. The old rats were retired breeders obtained at approximately 9 months of age and housed in a pathogen-free facility. Previous work in our laboratory has indicated that breeding history is not an important determinant in the expression of spatial learning deficits. The results obtained from this previous work showed that middle-aged (12-month-old) retired breeders were not impaired in either place or cue learning when compared with virgin young (4-6month-old) rats (Rapp et al., 1987). All of the rats were housed individually in an environment with a 12 -h light/dark cycle and a temperature of $25^{\circ} \mathrm{C}$. The rats were allowed food (Wayne Lab Blox) and water ad lib. All experiments were conducted between 9:00 a.m. and 2:00 p.m.

Procedure. The water maze and its surrounding environment were the same as in Experiment 1. Training trials also were conducted as described in the first experiment. The training schedule, however, consisted of three trials per day for 6 days.

Probe trials were conducted on every sixth trial. During each probe trial, the platform was removed and the animal was allowed to swim freely for $30 \mathrm{sec}$. Inspection of the probe-trial data from the previous experiment indicated that animals spent most of their time in the training quadrant during the first $30 \mathrm{sec}$ of the 90 -sec probe trials. Escape latencies, quadrant time, and annulus crossings were measured as in Experiment 1. In addition, for Trials 13-17, path length and heading error were analyzed from videotapes. Heading error was defined as the angle of deviation from the most direct path between the entry point and the escape platform, where the vertex of the angle was $18 \mathrm{~cm}$ from the maze wall.

Statistical analysis. Escape latencies were analyzed by a oneway ANOVA with repeated measures. Heading errors and path lengths were analyzed using a $t$ test. Quadrant time and annulus crossings were analyzed using a two-way between-groups ANOVA (age $\times$ quadrant) with repeated measures (probe trial number). All post hoc comparisons of group means were conducted with the Newman-Keuls statistic.

\section{Results}

The data presented in Figure 6 indicate that although aged and young animals began training with similar escape latencies, young animals decreased their latencies to locate the escape platform at a more rapid rate than did aged animals. Analysis of these data revealed significant main effects of age $[F(1,18)=13.83 ; p<.001]$ and trials $[F(14,252)=3.89 ; p<.001]$. Subsequent comparisons of group means across trials showed that a significant difference in the performance of young and aged rats did not appear until Trial $6(p<.03)$. Furthermore, significant within-group training effects were evident for young animals during the first five trials $(p<.01)$, but not for aged animals.

Analysis of the path lengths for the final five trials (Trials 13-17) revealed that old animals took longer routes to reach the escape platform than did young animals $(p<.01)$. These data are presented in Table 1. The increased path length observed in aged animals, however,

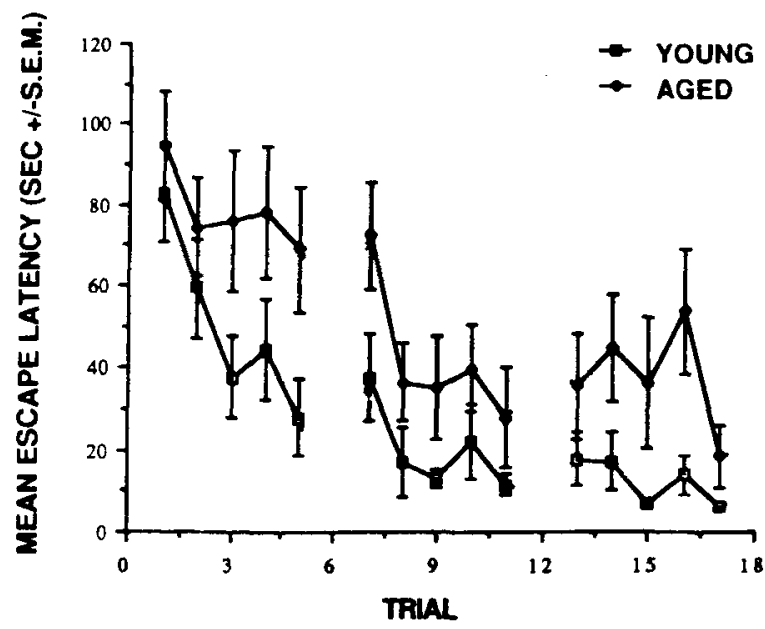

Figure 6. Mean escape latencies of young and aged animals for Trials 1-17. The escape latencies of aged animals are indicated by circles and the latencies of young animals are represented by squares. Probe trials were interpolated on Trials 6, 12, and 18. They are indicated by breaks in the graph. 
Table 1

Mean Path Length (in Meters) and Heading Errors (Both $\pm S E M$ ) for Aged and Young Rats

\begin{tabular}{lcc}
\hline & Path Length & Heading Error \\
\hline Young & $2.85 \pm 0.48^{*}$ & $29.72^{\circ} \pm 3.75^{\circ}$ \\
Aged & $5.52 \pm 0.92$ & $37.22^{\circ} \pm 5.19^{\circ}$ \\
\hline
\end{tabular}

*Differs significantly from aged $(p<.01)$.

was not entirely due to errors in initial heading angle. Heading-error analysis did not reveal a significant agerelated difference at this point in training, although heading error in aged animals was higher and more variable than in young animals. These results indicate that aged animals take a more circuitous route to the escape platform than do their young counterparts. Observation of the aged animals at this point confirmed the impression that they were actively engaged in a search for the escape platform, but that their search pattern was not as precise as that exhibited by young animals.

The data represented in Figures $7 \mathrm{a}$ and $7 \mathrm{~b}$ indicate that by the end of training, aged animals still exhibited less spatial bias for the training quadrant than did young animals. An analysis of quadrant time revealed a significant quadrant effect $[F(3,116)=14.88 ; p<.001]$, due to an overall bias for the former training quadrant $(p<.01)$. There was also a significant age $\times$ quadrant interaction $[F(3,116)=3.75 ; p<.013]$, along with a significant age $\times$ quadrant $\times$ probe trial interaction $[F(6,232)=2.15 ; p<.049]$. A simple-effects analysis indicated that an age difference in the allocation of swimming time was apparent only for the training quadrant $(p<.01)$. Further analysis of simple effects indicated that young animals showed a significant bias for their former training quadrant on each probe trial $(p<.01)$. In addition, the amount of time young animals spent in the training quadrant increased significantly across probe trials $(p<.03)$. In contrast, old animals did not spend significantly more time in their former training quadrant relative to the other quadrants on any probe trial. Old animals did, however, increase the amount of time in their former training quadrant across probe trials $(p<.05)$.

Analysis of the annulus-crossings measure yielded significant age $[F(1,68)=11.02 ; p<.001]$, quadrant $[F(3$, $68)=11.15 ; p<.001]$, and trial $[F(2,136)=5.48$; $p<.005]$ effects, as well as a significant age $\times$ quadrant interaction $[F(3,68)=7.82 ; p<.001]$. Subsequent analysis of these effects showed that young animals crossed over the position that had contained their escape platform more often than did aged animals $(p<.01)$. Furthermore, whereas young animals demonstrated a significant bias for their training annulus $(p<.01)$ during each probe trial $(p<.01)$, old animals did not show a similarly significant bias during any probe trial. This can be seen by comparing Figures $8 \mathrm{a}$ and $8 \mathrm{~b}$.

\section{Discussion}

The impaired performance of the aged animals in this experiment was similar to that previously reported for place learning in the water maze (Biegon et al., 1986; Decker et al., in press; Gage \& Bjorklund, 1986; Gage et al., 1984; Rapp et al., 1987). Aged animals had longer escape latencies during training and used longer, more circuitous paths to reach the escape platform, indicating that they use a less efficient search strategy than do young animals. Performance on the interpolated probe trials also indicated that aged animals required more training to achieve any spatial bias, and never reached the degree of spatial bias observed in young animals.

The configuration of stimuli provided by the maze environment in these experiments was designed to approximate the simplicity and salience that are typical of cues used in visual discrimination tasks or cue learning: tasks that aged rats learn as well as do young rats (Kay \& Sime, 1962; Lowy et al., 1985; Rapp et al., 1987; Sarter \& Markowitsch, 1983). Nonetheless, the results obtained

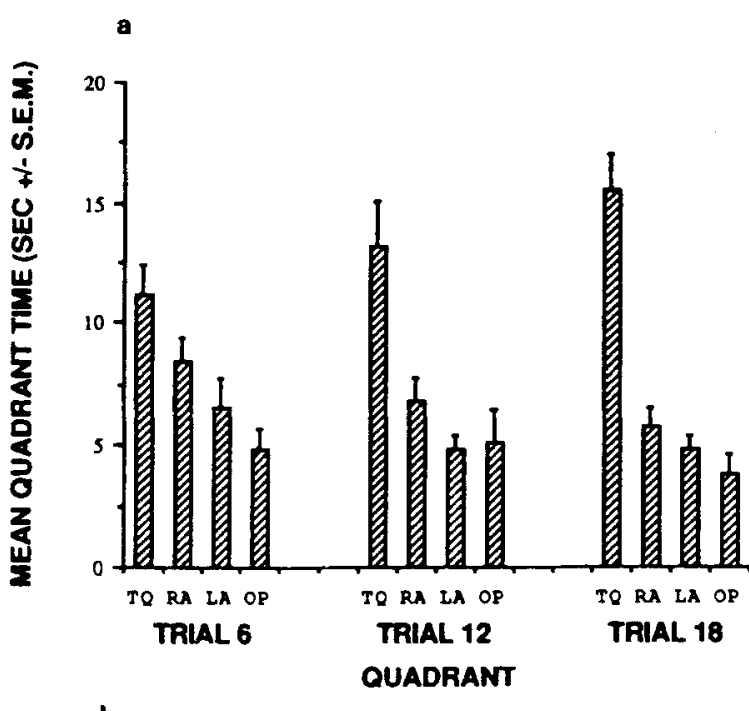

b

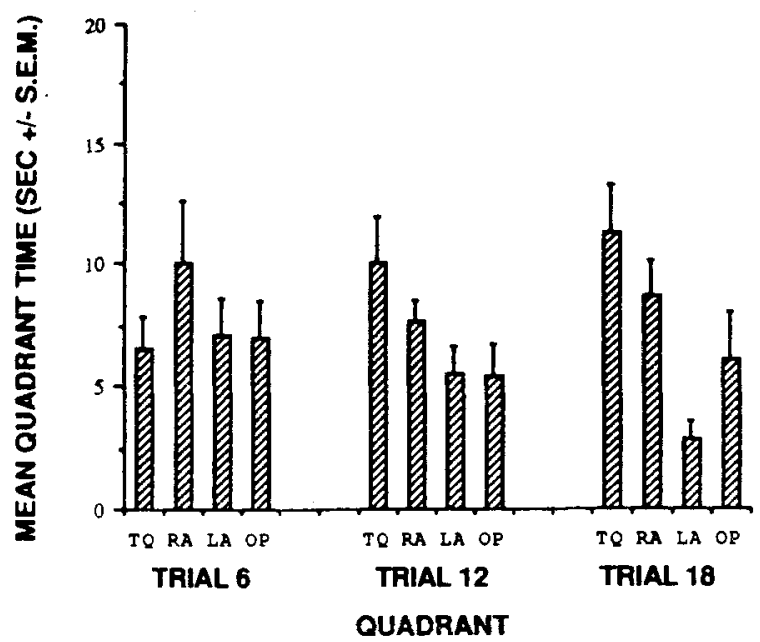

Figure 7. Mean quadrant time during Probe Trials 6, 12, and 18. a: Data for young animals. b: Data for aged animals. $T Q=$ former training quadrant, $\mathbf{R A}=$ right adjacent, $L A=$ left adjacent, and $O P=$ quadrant opposite the training quadrant. 
$\mathbf{a}$

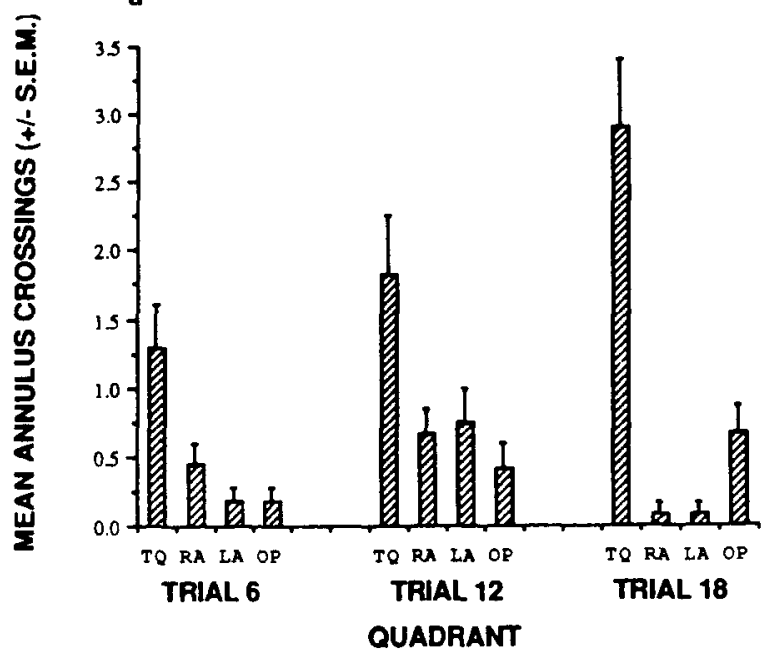

b

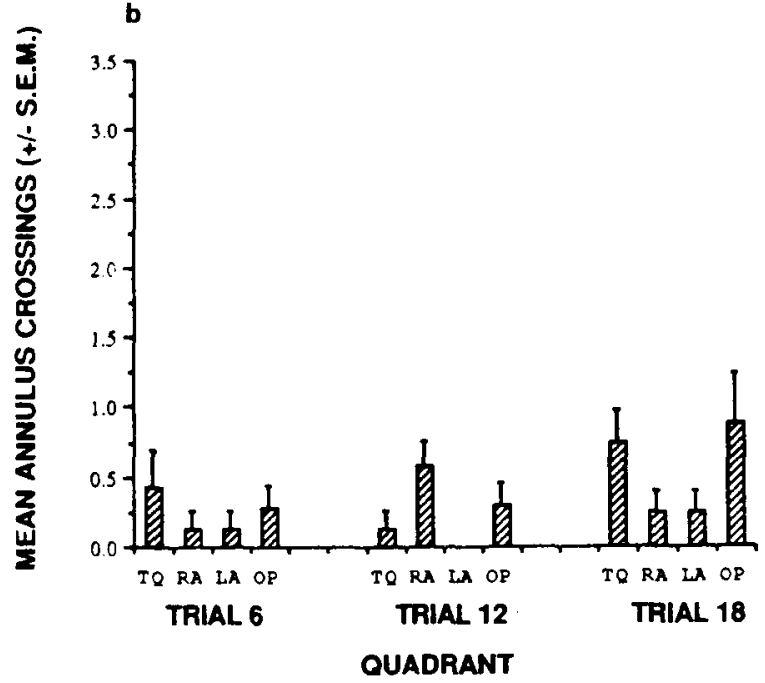

Figure 8. Mean number of annulus crossings during Probe Trials 6,12 , and 18. a: Data for young animals. b: Data for aged animals. $\mathbf{T Q}=$ former training quadrant, $\mathbf{R A}=$ right adjacent, $\mathbf{L A}=$ left adjacent, and $O P=$ quadrant opposite the training quadrant.

from the present study indicated that aged animals were impaired. Therefore, the place-learning deficit of aged rats that is frequently observed in the water maze using a traditional array of stimuli in the surrounding room may not be attributable to a subtle impairment in the ability to perceive such complex, and often distant, stimuli. The results obtained in the present experiment could, instead, suggest that aged animals may be impaired in their ability to perform a more specialized cognitive operation that is required to precisely locate a place based on a configuration of stimuli. Such an interpretation is at least consistent with the behaviorial impairment observed in aged animals in many different spatial tasks, and it remains a viable interpretation for further study.

Our findings are interesting in light of data obtained in another laboratory that suggest that a similar arc/wall configuration can act as a polarizing stimulus for place- field formation in hippocampal complex spike cells (Muller, Kubie, \& Ranck, 1987). In this work, a contrasting color cue card that subtended a $100^{\circ}$ arc around the inside wall of a large cylindrical apparatus was used. Hippocampal complex spike cells fired reliably in response to the animal's location relative to the arc. Rotation of the arc frequently resulted in a compensatory rotation of a cell's place field, whereas removal of the arc usually resulted in unpredictable angular rotations of the place field and some loss of spatial coherence in the cell's firing (Muller \& Kubie, 1987). Our data have provided a behavioral parallel to these findings in that rotation of the arc produced a corresponding rotation in the animal's search for the escape platform and removal of the arc produced some apparent loss in the accuracy of the search pattern.

Barnes and her colleagues have linked the impaired spatial learning ability of aged rats to deficits in hippocampal function. For example, aged rats with impaired acquisition of the holeboard escape task also show slower development of long-term enhancement (LTE) and a more rapid rate of LTE decay than do young animals (Barnes \& McNaughton, 1985). Furthermore, aged animals show decreased place-field specificity and less reliable placecell firing (Barnes, McNaughton, \& O'Keefe, 1983). The findings that hippocampal place fields are formed in the presence of a configuration of stimuli similar to that used in our experiments, and that hippocampal place fields are less specific in aged animals, suggests that the hippocampal circuitry involved in spatial mapping may be impaired in aged animals. Such an impairment may provide a neurobiological substrate for the emergence of spatial learning deficits during aging.

\section{REFERENCES}

BarNes, C. A. (1979). Memory deficits associated with senescence: A neurophysiological and behavioral study in the rat. Joumal of Comparative \& Physiological Psychology, 93, 74-104.

Barnes, C. A., \& MCNaughton, B. L. (1985). An age comparison of the rates of acquisition and forgetting of spatial information in relation to long-term enhancement of hippocampal synapses. Behavioral Neuroscience, 99, 1040-1048.

Barnes, C. A., MCNaughton, B. L., \& O'KeEFe, J. (1983). Loss of place specificity in hippocampal complex-spike cells in the senescent rat. Neurobiology of Aging, 4, 113-119.

Barnes, C. A., NAdel, L., \& Honig, W. K. (1980). Spatial memory deficit in senescent rats. Canadian Journal of Psychology, 34, 29-39.

Biegon, A., Greenberger, V., \& Segal, M. (1986). Quantitative histochemistry of brain acetylcholinesterase and learning rate in the aged rat. Neurobiology of Aging, 7, 215-217.

Davis, H. P., IDowu, A., \& Gibson, G. E. (1983). Improvement of 8-arm maze performance in aged Fisher 344 rats with 3,4diaminopyridine. Experimental Aging Research, 9, 211-214.

Decker, M. W., Pelleymounter, M. A., \& Gallagher, M. (in press). The effects of training on a spatial memory task on high affinity choline uptake in hippocampus and cortex in young adult and aged rats. Journal of Neuroscience.

deToledo-Morrell, L., Morrell, F., \& Fleming, S. (1984). Agerelated deficits in spatial memory are related to impaired hippocampal kindling. Behavioral Neuroscience, 98, 902-907. 
deToledo-Morrell, L., Morrell, F., Fleming, S., \& Cohen, M. (1984). Pentoxifylline reverses age related deficits in spatial memory. Behavioral \& Neural Biology, 42, 1-8.

GAGE, F. H., \& BJoRkLund, A. (1986). Cholinergic septal grafts into the hippocampal formation improve spatial learning and memory in aged rats by an atropine-sensitive mechanism. The Journal of Neuroscience, 6, 2837-2847.

GAGE, F. H., DunNeTt, S. S., \& BJorklund, A. (1984). Spatial learning and motor deficits in aged rats. Neurobiology of Aging, 5, 43-48.

Gallagher, M., Bostock, E., \& KING, R. (1985). Effects of opiate antagonists on spatial memory in young and aged rats. Behavioral \& Neural Biology, 44, 374-385.

KAY, H., \& SIME, M. E. (1962). Discrimination learning with old and young rats. Journal of Gerontology, 17, 75-80.

Lowy, A., Ingram, D., Olton, D., Waller, S., Reynolds, M., \& LONDON, E. (1985). Discrimination learning requiring different memory components in rats: Age and neurochemical comparisons. Behavioral Neuroscience, 99, 638-651.

MoRRIS, R. G. (1981). Spatial localization does not require the presence of local cues. Leaming \& Motivation, 12, 239-260.
Muller, R. U., \& KuBie, J. L. (1987). The effects of changes in the environment on the spatial firing of hippocampal complex-spike cells. Journal of Neuroscience, 7, 1951-1968.

Muller, R. U., Kubie, J. L., \& RANCK, J. B., JR. (1987). Spatial firing patterns of hippocampal complex-spike cells in a fixed environment. Journal of Neuroscience, 7, 1935-1951.

Rapp, P. R., Rosenberg, R. A., \& Gallagher, M. (1987). An evaluation of spatial information processing in aged rats. Behavioral Neuroscience, 101, 3-12.

Sarter, M., \& Markowitsch, H. (1983). Reduced resistance to progressive extinction in senescent rats: A neuroanatomical and behavioral study. Neurobiology of Aging, 4, 203-215.

Wallace, J. E., Krauter, E. E., \& CampBell, B. A. (1980). Animal models of declining memory in the aged: Short-term and spatial memory in the old rat. Journal of Gerontology, 35, 355-363.

(Manuscript received March 23, 1987; revision accepted for publication July $1,1987$. 